\title{
UNRAMIFIED COHOMOLOGY OF FINITE GROUPS OF LIE TYPE
}

by

\author{
Fedor Bogomolov, Tihomir Petrov and Yuri Tschinkel
}

\begin{abstract}
We prove vanishing results for unramified stable cohomology of finite groups of Lie type.
\end{abstract}

\section{Contents}

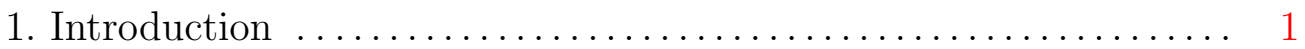

2. Equivariant birational geometry $\ldots \ldots \ldots \ldots \ldots \ldots \ldots \ldots \ldots \ldots$

3. Equivariant birational geometry of classical groups $\ldots \ldots \ldots \ldots 8$

4. Stable cohomology ................................ 12

5. Comparison with Serre's negligible classes ............... 15

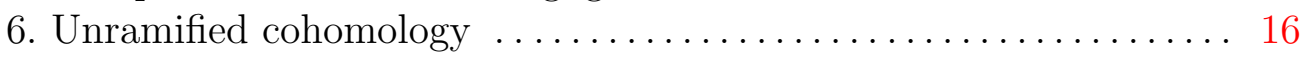

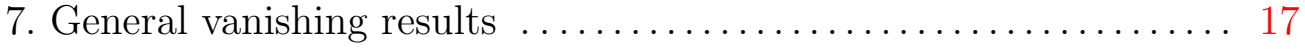

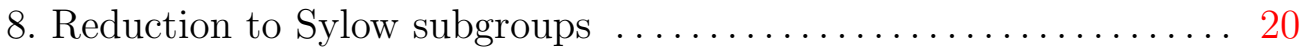

References ............................................. 22

\section{Introduction}

Let $k$ be an algebraically closed field, $G$ a finite group and $V$ a faithful representation of $G$ over $k$. In this note we compute cohomological obstructions to stable rationality of quotients of $V$ by $G$ introduced by Saltman [13] and [4] and studied in [8], [11], [5].

KEY WORDS AND PHRASES. — Rationality, finite simple groups, unramified cohomology. 
Let $K=k(V)^{G}$ be the function field of the quotient variety and

$$
s: \mathcal{G}_{K} \rightarrow G
$$

the natural homomorphism from the absolute Galois group of $K$ to $G$. We have an induced map on cohomology with coefficients in the torsion group $\mathbb{Z} / \ell$, with trivial $G$-action,

$$
s_{i}^{*}: H^{i}(G, \mathbb{Z} / \ell) \rightarrow H^{i}\left(\mathcal{G}_{K}, \mathbb{Z} / \ell\right) .
$$

Note that $s_{i}^{*}$ depends on the ground field $k$, but not on the choice of the faithful representation $V$ over that field. The groups

$$
H_{k, s}^{i}(G, \mathbb{Z} / \ell):=H^{i}(G, \mathbb{Z} / \ell) / \operatorname{Ker}\left(s_{i}^{*}\right),
$$

are called stable cohomology groups over $k$. They form a finite ring. We may consider them as subgroups of $H^{i}\left(\mathcal{G}_{K}, \mathbb{Z} / \ell\right)$. Every divisorial valuation $\nu \in \mathrm{Val}_{K}$ of $K$ defines a residue map

$$
\partial_{\nu}: H^{i}\left(\mathcal{G}_{K}, \mathbb{Z} / \ell\right) \rightarrow H^{i-1}\left(\mathcal{G}_{K_{\nu}}, \mathbb{Z} / \ell\right),
$$

where $\boldsymbol{K}_{\nu}$ is the residue field of $\nu$. The groups

$$
H_{k, u n}^{i}(G, \mathbb{Z} / \ell):=\bigcap_{\nu \in \operatorname{Val}_{K}} \operatorname{Ker}\left(\partial_{\nu} \circ s_{i}^{*}\right) \subset H_{k, s}^{i}(G, \mathbb{Z} / \ell)
$$

form a subring of $H_{k, s}^{*}(G, \mathbb{Z} / \ell)$. A basic fact is that if there exists a faithful representation $V$ of $G$ over $k$ and a unirational parametrization of the quotient $V / G$ whose degree is prime to $\ell$, then

$$
H_{k, u n}^{i}(G, \mathbb{Z} / \ell)=0, \text { for all } i>0 .
$$

In particular, these cohomology groups vanish if this quotient is stably rational.

For example, the rings of invariants of finite groups generated by pseudoreflections are polynomial, the corresponding quotient varieties rational, and the cohomological invariants trivial. In particular, all Weyl groups $\mathcal{W}$ of semisimple Lie groups have

$$
H_{k, \text { un }}^{i}(\mathcal{W}, \mathbb{Z} / \ell)=0, \text { for all } i>0, \text { and all } k .
$$

Conjecture 1.1. - Let $G$ be a finite simple group. Then

$$
H_{k, \text { un }}^{i}(G, \mathbb{Z} / \ell)=0, \text { for all } i>0 \text {, all } k \text { and all } \ell \text {. }
$$


The $i=2$ case of this conjecture was proved for $G=\mathrm{PSL}_{n}\left(\mathbb{F}_{q}\right)$ and $k=\mathbb{C}$ in [5] and for simple and quasi-simple groups of Lie type in [10]. Examples of functions fields with vanishing second and nonvanishing third unramified cohomology were given in [12]. Here we prove that many of these cohomology groups vanish for finite groups of Lie type, for $k=\overline{\mathbb{F}}_{q}$. In fact, we prove the stable rationality of many associated quotients spaces. Our main theorem is:

TheOREm 1.2. - Let $G$ be one of the following groups

$$
\mathrm{SL}_{n}\left(\mathbb{F}_{q}\right), \quad \mathrm{Sp}_{2 n}\left(\mathbb{F}_{q}\right),
$$

or their twisted forms

$$
{ }^{2} \mathrm{SL}_{n}\left(\mathbb{F}_{q}\right), \quad{ }^{2} \mathrm{Sp}_{2 n}\left(\mathbb{F}_{q}\right) .
$$

Let $V$ be a faithful representation of $G$ over $k=\overline{\mathbb{F}}_{p}$. Then the quotient of $V$ by $G$ is stably rational over $k$.

In particular, Conjecture 1.1 holds in these cases for $\ell \nmid q$ and $k=\overline{\mathbb{F}}_{p}$. Our main tool is a theorem of Lang which proves the rationality of certain quotient spaces over $\overline{\mathbb{F}}_{p}$.

THEOREM 1.3. - Let $\mathrm{G}$ be a semi-simple simply-connected Lie group defined over a finite field $\mathbb{F}_{q}$. Then the image

$$
H^{i}\left(\mathrm{G}\left(\mathbb{F}_{q}\right), \mathbb{Z} / \ell\right) \rightarrow H_{k, s}\left(\mathrm{G} / \mathrm{G}\left(\mathbb{F}_{q}\right), \mathbb{Z} / \ell\right)
$$

is zero, for $k=\overline{\mathbb{F}}_{q}$, all $i>0$ and $\ell \nmid q$.

Combining this with results of Tits [16] we obtain the following:

THEOREM 1.4. - Let $G$ be a finite quasi-simple group of Lie type over a finite field of characteristic p. Put

$$
d(G):= \begin{cases}\{p\} & \text { if } G \text { is of type } A_{n}, B_{n}, C_{n}, D_{n} \text { or } G_{2} \\ \{2,3, p\} & \text { if } G \text { is of type } F_{4}, E_{6}, E_{7} ; \\ \{2,3,5, p\} & \text { if } G \text { is of type } E_{8} .\end{cases}
$$

Then for all algebraically closed fields $k$ one has

$$
H_{k, u n}^{i}(G, \mathbb{Z} / \ell)=0, \text { for all } i>0 \text {, and all } \ell \notin d(G) .
$$

For $k=\overline{\mathbb{F}}_{p}$ the vanishing also holds for $\ell=p$. 
Here is the roadmap of the paper. In Section 2 we study the birational type of quotients $G \backslash \mathrm{G} / H$, where $\mathrm{G}$ is an algebraic group over an algebraically closed field $k$ and $G, H \subset \mathrm{G}(k)$ are finite subgroups, acting on $\mathrm{G}$ by translations on the left, resp. on the right. In Section 3 we study the classical groups. In Section 4 we introduce stable and unramified cohomology over arbitrary algebraically closed fields and prove their basic properties. In Section 7 we establish general vanishing results, applying theorems of Lang and Tits. In Section 8 we sketch another approach which is using the structure of Sylow subgroups of quasisimple groups of Lie type. As an example we prove the triviality of unramified cohomology over $\mathbb{C}$ for all groups $G L_{n}\left(\mathbb{F}_{q}\right)$ and $\ell$ coprime to $q$.

Acknowledgments: The first author was supported by NSF grant DMS0701578 and the third author by NSF grants DMS-0554280 and DMS-0602333.

\section{Equivariant birational geometry}

We work over an algebraically closed field $k$. We say that $k$-varieties $X$ and $Y$ are stably birational, and write $X \sim Y$, if $X \times \mathbb{A}^{n}$ is birational to $Y \times \mathbb{A}^{m}$, for some $n, m \in \mathbb{N}$.

Let $G$ be an algebraic group and $X$ an algebraic variety over $k$, with a $G$-action

$$
\lambda: G \times X \rightarrow X .
$$

We will sometimes consider different actions of the same group. To emphasize the action we will write $\lambda(G) \backslash X$ for the quotient of $X$ by the $\lambda$-action of $G$; we write $G \backslash X$, when the action is clear from the context.

We say that the action of $G$ is almost free if there exists a Zariski open subset $X^{\circ} \subset X$ on which the action is free. In particular, the quotient map $X \rightarrow G \backslash X$ is separable.

ExAmple 2.1. - Let $V$ be a faithful complex representation of $G$. Then $G$ acts almost freely on $V$.

LEMma 2.2. - Let $G$ be a finite group and $V$ a faithful representation of $G$ over an algebraically closed field $k$. Let $Y$ be an affine variety over $k$, with a free $G$-action, and $y \in Y(k)$ a point. 
For every Zariski open $U \subset V$ there exist a $G$-equivariant $k$-morphism $\phi_{U}$ : $Y \rightarrow V$ and a Zariski open $G$-invariant subset $Y^{\circ} \subset Y$ such that

$-y \in Y^{\circ}(k)$;

$-\phi_{U}\left(Y^{\circ}\right) \subset U$.

Proof. - It suffices to consider $V:=\mathbb{A}_{k}^{|G|}$, the affine $k$-space, with the induced faithful $G$-action. For any divisor $D \subset V$ there exists a Zariski open subset $U \subset V$ such that for every point $v \in U(k)$ its $G$-orbit $G \cdot v \notin D$. For every Zariski open $U \subset V$ there exists a $G$-equivariant $k$-morphism $\phi_{U}: Y \rightarrow V$ such that $\phi_{U}(y) \in U(k)$ (functions separate points). This implies the existence of a Zariski open $G$-invariant subset $Y^{\circ} \subset Y$ with the claimed properties.

A $G$-variety $X$ is called $G$-affine, and the corresponding action affine, if there exists a $G$-equivariant birational isomorphism between $X$ and a faithful representation of $G$. Let $\mathcal{V}$ and $X$ be affine $G$-varieties. A $G$-morphism $\pi$ : $\mathcal{V} \rightarrow X$ is called an affine $G$-bundle if it is an affine bundle over some open subset $X^{\circ} \subset X$ and the $G$-action is compatible with this structure of an affine bundle.

By Hilbert 90 , an affine $G$-bundle $\mathcal{V} \rightarrow X$ is $G$-birational to a finite dimensional $G$-representation over the function field of $K=k(X)$, compatible with the given $G$-action on $K$. A morphism $\rho: X \rightarrow B$ of $G$-varieties will be called a $G$-ruling (and $X-G$-ruled) over $B$ if there exists a finite set of affine $G$-varieties

$$
X_{n}=X, B_{n-1}, X_{n-1}, B_{n-2}, X_{n-2}, \ldots, X_{1}, B_{0}=B
$$

such that $X_{i} \rightarrow B_{i-1}$ is an affine $G$-bundle and $B_{i} \subset X_{i}$ a $G$-stable Zariski open subset, for $i=1, \ldots, n$.

Lemma 2.3. - Assume that $\rho: X \rightarrow B$ is a G-ruling over $B$ and that the action of $G$ on $X$ is almost free. Then $X$ is $G$-affine.

Proof. - Follows from Hilbert 90.

Let $X, Y$ be smooth varieties with an almost free action of $G$. We write $X \stackrel{G}{\rightsquigarrow} Y$ if there exist a $G$-representation $V$, a Zariski open $G$-stable subset $X^{\circ} \subset X$ and a $G$-morphism (not necessarily dominant) $\beta: X^{\circ} \times V \rightarrow Y$. We write $X \stackrel{G}{\leftrightarrow} Y$, and say that the $G$-actions are equivalent, if $X \stackrel{G}{\rightsquigarrow} Y$ and $Y \stackrel{G}{\rightsquigarrow} X$. 
LEMmA 2.4. - If $X \stackrel{G}{\rightsquigarrow} Y$, then the morphism

$$
\beta: G \backslash(X \times Y) \rightarrow G \backslash X
$$

has a rational section.

Proof. - Consider the morphism

$$
\beta^{\prime}: G \backslash(X \times V \times Y) \rightarrow G \backslash X,
$$

where $G$ acts diagonally. The graph of the map $X \rightarrow(Y \times V)$ is $G$-stable and gives a section of $\beta^{\prime}$. The projection of this section to $G \backslash(X \times Y)$ is a section of $\beta$.

LEMmA 2.5. - Let $\mathrm{G}$ be a Lie group over an algebraically closed field $k$. Let $G \subset \mathrm{G}(k)$ be a finite subgroup. Let $X$ be an algebraic variety over $k$ with an almost free action of $G$. Assume that $X \stackrel{G}{\rightsquigarrow} \mathrm{G}$, where $\mathrm{G}$ is considered as a $G$-variety, with a left action. Then

$$
G \backslash(X \times \mathrm{G}) \sim G \backslash X .
$$

Proof. - By Lemma 2.4, there is a Zariski open $G$-stable subset $X^{\circ} \subset X$ so that the $G$-morphism (projection to the first factor)

$$
\beta: G \backslash(X \times \mathrm{G}) \rightarrow(G \backslash X)
$$

has a section. We also have a right action of $\mathrm{G}$, which preserves the fibration structure given by $\beta$. Thus it is a principal homogeneous space over $G \backslash X^{\circ}$, for some $G$-stable Zariski open $X^{\circ} \subset G$, with a section. Hence it is birational to $(G \backslash X) \times \mathrm{G}$. It suffices to recall that $\mathrm{G}$ is rational over $k$.

Let $\mathrm{G}$ be a connected algebraic group and $F \in \operatorname{Aut}_{k}(\mathrm{G})$ a $k$-automorphism of $\mathrm{G}$. Let $G \subset \mathrm{G}(k)$ be a finite subgroup, with a natural left action

$$
\begin{aligned}
\lambda: G \times G & \rightarrow G \\
(\gamma, g) & \mapsto \gamma \cdot g
\end{aligned}
$$

We also have an $F$-twisted right action

$$
\begin{aligned}
& \rho^{F}: G \times \mathrm{G} \rightarrow \quad \mathrm{G} \\
& (\gamma, g) \mapsto g \cdot F\left(\gamma^{-1}\right)
\end{aligned}
$$

and an $F$-conjugation

$$
\begin{aligned}
& \kappa^{F}: G \times \mathrm{G} \rightarrow \quad \mathrm{G} \\
& (\gamma, g) \mapsto \gamma \cdot g \cdot F\left(\gamma^{-1}\right) .
\end{aligned}
$$


LEMma 2.6. - Assume that $G \subset \mathrm{G}(k)$ has the following properties:

(1) there exists a faithful $G$-representation $V$ such that $V \stackrel{G}{\rightsquigarrow} \mathrm{G}$, where $G$ acts on $\mathrm{G}$ via $\lambda$;

(2) the twisted action $\rho^{F}$ on $\mathrm{G}$ is almost free.

Then the quotient of $\mathrm{G}$ by the F-twisted conjugation $\kappa^{F}$ of $G$ is stably birational to the quotient of $\mathrm{G}$ by $\lambda$.

Proof. - Consider the diagonal action of $G$ on $\mathrm{G} \times \mathrm{G}$ :

$$
\begin{aligned}
& G \times \mathrm{G} \times \mathrm{G} \stackrel{\left(\lambda, \rho^{F}\right)}{\longrightarrow} \quad \mathrm{G} \times \mathrm{G} \\
& \left(\gamma, g, g^{\prime}\right) \quad \mapsto \quad\left(\gamma \cdot g, g^{\prime} \cdot F\left(\gamma^{-1}\right)\right) .
\end{aligned}
$$

Let $\Delta^{F}:=\left\{\left(g, F^{-1}(g)\right)\right\} \subset \mathrm{G} \times \mathrm{G}$ be the $F$-twisted (anti)diagonal. Then $\Delta^{F}$ is preserved under the $\left(\lambda, \rho^{F}\right)$-action of $G$ and descends to a section of the principal (right) G-fibration

$$
\left(\lambda, \rho^{F}\right)(G) \backslash(\mathrm{G} \times \mathrm{G}) \rightarrow \rho^{F}(G) \backslash \mathrm{G},
$$

projection to the second factor. It follows that

$$
\rho^{F}(G) \backslash \mathrm{G} \sim\left(\lambda, \rho^{F}\right)(G) \backslash(\mathrm{G} \times \mathrm{G}) .
$$

Observe that

$$
\rho^{F}(G) \backslash G \sim \lambda(G) \backslash G
$$

Now we show that

$$
\kappa^{F}(G) \backslash \mathrm{G} \sim\left(\lambda, \rho^{F}\right)(G) \backslash(\mathrm{G} \times \mathrm{G}) .
$$

Let $V$ be a faithful representation of $G$ as in (1) and $V^{\circ} \subset V$ a $G$-stable Zariski subset admitting a $G$-map into $G$, considered with the $\lambda$-action of $G$. We know that there exists a $G$-morphism $\xi: G \rightarrow V$, where $G$ is considered with the $\kappa^{F}$-action of $G$, such that $\xi(\mathrm{G}) \cap V^{\circ} \neq \emptyset$ (see Lemma 2.2). It follows that $\mathrm{G} \stackrel{G}{\rightsquigarrow} \mathrm{G}$, where the source carries the $\kappa^{F}$-action of $G$ and the image the $\lambda$-action of $G$. Equation (2.1) now follows from Lemma 2.4.

Corollary 2.7. - Let $G \subset \mathrm{G}(k)$ by a finite subgroup satisfying Assumption (1) of Lemma 2.6. Let $V$ be a faithful representation of $G$ over $k$. Then

$$
G \backslash G \sim G \backslash V .
$$

More generally, for any $X$ with an almost free action of $G$ we have

$$
G \backslash(X \times V) \sim G \backslash X .
$$


Proof. - Note that $G \backslash(\mathrm{G} \times V)$ is a vector bundle over $G \backslash \mathrm{G}$, and hence stably birational to it. On the other hand, it is a right G-fibration over $G \backslash V$ with section defined by the $G$-equivariant map $V \rightarrow \mathrm{G}$.

To prove the statement for $X$ it suffices to notice that a dense Zariski open $G$-stable subset $X^{\circ} \subset X$ admits a nontrivial $G$-morphism to $V$.

\section{Equivariant birational geometry of classical groups}

In this section, $k$ is an algebraically closed field, of any characteristic.

Lemma 3.1. - The conjugation action $\kappa: \mathrm{SL}_{2} \rightarrow \mathrm{SL}_{2}$ is equivalent to a linear action.

Proof. - Realize $\mathrm{SL}_{2}$ as a nonsingular quadric in $\mathbb{A}^{4}=\mathrm{M}_{2}$. The conjugation action is linear on $\mathrm{M}_{2}$ and has a fixed point corresponding to the identity. The projection of $\mathrm{SL}_{2}$ from the identity to the locus of trace zero matrices is equivariant and has degree 1 . Hence the conjugation action is rationally equivalent to the action on trace zero matrices.

Lemma 3.2. - Let $G=(\mathbb{Z} / 2)^{n}$ and $X$ be a $G$-affine variety over $k$. Then $G \backslash X$ is rational.

LEMMA 3.3. - Let $G \subset \mathrm{PGL}_{2}(k) \simeq \mathrm{SO}_{3}(k) \subset \mathrm{M}_{2}(k)$ be a finite subgroup. Then the action of $G$ on $\mathrm{PGL}_{2}$ by conjugation is equivalent to the left (linear) action of $G$ on trace zero matrices $\mathrm{M}_{2}^{0} \subset \mathrm{M}_{2}$. In particular, $G \backslash \mathrm{PGL}_{2}$ is stably rational.

LEMma 3.4. - The left action of $(\mathbb{Z} / 2)^{2} \subset \mathrm{SO}_{3} \hookrightarrow \mathrm{SO}_{4}$ is linear.

Proof. - Consider the subgroup $(\mathbb{Z} / 2)^{3} \subset \mathrm{SO}_{4}$. It contains a central subgroup $\mathbb{Z} / 2$ and a complementary subgroup $H_{2}=(\mathbb{Z} / 2)^{2} \subset \mathrm{SO}_{3}=\mathrm{PGL}_{2}$. By Lemma 3.1, the conjugation action $H_{2} \subset \mathrm{PGL}_{2}$ is linear.

This is a subgroup of the diagonal subgroup $\mathrm{SO}_{3}=\mathrm{PGL}_{2} \subset \mathrm{SL}_{2} \times \mathrm{SL}_{2} /(\mathbb{Z} / 2)$. Note that $\mathrm{SO}_{4}$ is a product of $\mathrm{SO}_{3}=(h, h)$ and $\operatorname{Spin}_{3}=(g, 1)$, and that conjugation by elements in $(\mathbb{Z} / 2)^{2}$ respects this decomposition. Thus the action is a vector bundle over $\mathrm{SL}_{2} /\left(\mathrm{Q}_{8}\right)_{\text {conj }}=\mathrm{SL}_{2} /(\mathbb{Z} / 2)^{2}$ (where $\mathrm{Q}_{8}$ are the quaternions).

Hence the action on $\mathrm{SO}_{4}$ is linear and the automorphism $F$ is the identity on the diagonal $\mathrm{SO}_{3}$. The same holds for the twisted $F$-action. 
Lemma 3.5. - Let $\mathrm{G}$ be an algebraic group over $k$. Assume that $\mathrm{G}$ admits an affine action on itself, e.g., $\mathrm{G}=\mathrm{GL}_{n}, \mathrm{SL}_{n}, \mathrm{Sp}_{n}$. Let $G \subset \mathrm{G}(k)$ be a finite subgroup which has trivial intersection with the center of $\mathrm{G}$.

Then the conjugation action of $G$ on $G$ is stably birationally equivalent to a linear action.

Proof. - By Corollary 2.7, the diagonal left translation action of $G$ on $\mathrm{G} \times \mathrm{G}$ is equivalent to the action on a principal G-bundle over $\mathrm{G}$, with $G$ acting on the base by conjugation. This proves the equivalence.

Corollary 3.6. - Let $G \subset \mathrm{G}(k)$ be a finite subgroup as in Lemma 3.5. Assume that the action of $G$ on $\mathrm{G}$ by left translations is affine. Then both the left and the conjugation action of $G$ on $\mathrm{G}$ are stably birational to a linear action.

Proposition 3.7. - Let $\mathrm{G}$ be a classical simply-connected Lie group of type A or $C$, i.e., $\mathrm{G}=\mathrm{SL}_{n}$ or $\mathrm{G}=\mathrm{Sp}_{2 n}$ over $k$. Let $G \subset \mathrm{G}(k)$ be a finite subgroup. Then $\mathrm{G}$ is a $G$-affine variety, for the standard left action of $G$.

Proof. - Note first that any finite subgroup $G \subset \mathrm{GL}_{n}(k)$ induces a $G$-affine structure on $\mathrm{GL}_{n}$. Indeed, $\mathrm{GL}_{n} \subset \mathrm{M}_{n \times n}=\oplus_{i=1}^{n} V^{(i)}$, a direct sum of $n$ copies of the standard representation $V$ of $\mathrm{GL}_{n}$. Put $B_{0}=0$, a point, and $X_{1}:=V^{(1)}=$ $V$. We have a canonical projection $X_{1} \rightarrow B_{0}$. Define $X_{j} \subset \oplus_{i=1}^{j} V^{(i)}$ as the set of those vectors, whose projections to $\oplus_{i=1}^{j-1} V^{(i)}$ are linearly independent, and $B_{j} \subset X_{j}$ as the subset of vectors in $X_{j}$, which are linearly independent in $\oplus_{i=1}^{j} V^{(i)}$, under the standards identification $V=V^{(i)}$. For any $G \subset \mathrm{GL}_{n}$ this defines the structure of a $G$-ruling on $\mathrm{GL}_{n}$ over a point.

For $\mathrm{G}=\mathrm{SL}_{n}$, and $G \subset \mathrm{G}$ we have a similar $G$-ruling: For $j=1, \ldots, n-1$ it is the same as above. For $j=n$, put $X_{n}:=\mathrm{SL}_{n}$. The map $X_{n} \rightarrow B_{n-1}$ is the restriction of the map above. Explicitly, it is the projection to the first $(n-1)$-vectors $\left(v_{1}, \ldots, v_{n-1}\right)$, with fiber an affine subspace $F_{\left(v_{1}, \ldots, v_{n-1}\right)} \subset$ $V^{(n)}=V$, given by the affine equation in the coordinates of the last vector $\operatorname{det}\left(v_{1}, \ldots, v_{n}\right)=1$. Now we can apply Lemma 2.3 to conclude that $\mathbf{S L}_{n}$ is $G$-affine.

Note that by Tsen's theorem, the morphism $\mathrm{GL}_{n} \rightarrow \mathrm{GL}_{n} / \mathrm{SL}_{n}$ has a section. This gives a $G$-equivariant birational isomorphism $\mathrm{SL}_{n} \times \mathbb{G}_{m} \rightarrow \mathrm{GL}_{n}$. 
The group $\mathrm{G}=\mathrm{Sp}_{2 n}$ has a canonical embedding into $\mathrm{M}_{2 n \times 2 n}$, defined by the equations

$$
\omega\left(v_{i}, v_{i^{\prime}}\right)=\delta_{i^{\prime}, n+i}, \text { for } i<i^{\prime},
$$

( $\omega$ is the standard bilinear form and $\delta$ is the delta function). The system of projections is induced from the one above:

$$
X_{j}=\left\{\left(v_{1}, \ldots, v_{j}\right)\right\} \subset \oplus_{i=1}^{j} V^{(i)},
$$

satisfying equations (3.1), for indices $1 \leq i<i^{\prime} \leq j$, and the property that the vectors $v_{1}, \ldots, v_{j-1}$ are linearly independent in $V$, under the identifications $V^{(i)}=V$. The subvariety $B_{j} \subset X_{j}$ is given as the locus where $v_{1}, \ldots, v_{j}$ are linearly independent. Each map $X_{j} \rightarrow B_{j-1}$ is an affine $G$-bundle, for any finite subgroup $G \subset \mathrm{G}(k)$ - its fibers are given by a system of linear equations on the coordinates of $v_{j}$.

Proposition 3.8. - Let $\mathrm{G}=\mathrm{SO}_{n}$ and $G \subset \mathrm{G}(k)$ be a finite subgroup. Then there exist a $G$-ruling $X$, a variety $Y$ with trivial $G$-action and a $G$-equivariant finite morphism

$$
\pi: \mathrm{G} \times Y \rightarrow X
$$

Moreover, $\operatorname{deg}(\pi) \mid 2^{n-1}$.

Proof. - Keep the notations in the proof of Proposition 3.7: $\mathrm{G} \subset \mathrm{M}_{n}$.

Assume that $\operatorname{char}(k) \neq 2$. Every quadratic form can be diagonalized over $k$. Let $X_{n} \subset \mathrm{M}_{n}$ be the subvariety given by:

$$
\left(v_{i}, v_{i^{\prime}}\right)=\delta_{i^{\prime}, n+i}, \text { for } i<i^{\prime} .
$$

The system of projections is the same as above: $X_{j} \subset \oplus_{i=1}^{j} V^{(i)}$ is the subset of vectors satisfying equations (3.2), for indices $1 \leq i<i^{\prime} \leq j$, and the condition that $v_{1}, \ldots, v_{j-1}$ are linearly independent. The subvariety $B_{j} \subset X_{j}$ corresponds to $j$-tuples $\left(v_{1}, \ldots, v_{j}\right)$ which are linearly independent (as vectors in $\left.V=V^{(i)}\right)$. Each map $X_{j} \rightarrow B_{j-1}$ is a $G$-equivariant vector bundle, for any finite subgroup $G \subset \mathrm{G}(k)$. Each $X_{j}$ carries the action of a $j$-dimensional torus $\mathbb{G}_{m}^{j}$, over $k$, commuting with the action of $G$. The action of $\mathrm{G} \times \mathbb{G}_{m}^{n}$ on $X_{n}$ is transitive, and the stabilizer of a general $k$-point has order $2^{n-1}$. The claim follows, for $Y:=\mathbb{G}_{m}^{n}$.

Assume that $\operatorname{char}(k)=2$. In this case, $\mathrm{SO}_{2 n+1} \simeq \mathrm{Sp}_{2 n}$ and we can apply Proposition 3.7. We also have $\mathrm{SO}_{2 n} \subset \mathrm{Sp}_{2 n}$, where $\mathrm{Sp}_{2 n}(k)$ is the set of elements of $\mathrm{GL}_{2 n}(k)$ which preserve a symplectic bilinear form $\omega$, and $\mathrm{SO}_{2 n}(k)$ the set 
of those elements which in addition preserve a quadratic form $f$. The forms are related by the condition

$$
f(x+y)=f(x)+f(y)+\omega(x, y) .
$$

We may identify a general element $\gamma \in \mathrm{Sp}_{2 n}(k)$ with a choice of an orthogonal basis $\left\{v_{1}, \ldots, v_{2 n}\right\}$. Observe that the map

$$
\begin{aligned}
& \mathrm{Sp}_{2 n} \quad \rightarrow \quad \mathrm{Sp}_{2 n} / \mathrm{SO}_{2 n} \quad \sim \mathbb{A}^{2 n} \\
& \left\{v_{1}, \ldots, v_{2 n}\right\} \mapsto\left(f\left(v_{1}\right), \ldots, f\left(v_{2 n}\right)\right) .
\end{aligned}
$$

Indeed, $\gamma \in \mathrm{SO}_{2 n}(k)$ if and only if $f(\gamma x)=f(x)$, for all $x \in V$. We have

$$
\begin{aligned}
f\left(\sum_{i=1}^{2 n} a_{i} v_{i}\right) & =\sum_{i=1}^{2 n} a_{i}^{2} f\left(v_{i}\right)+\sum_{i \neq j} a_{i} a_{j}\left(v_{i}, v_{j}\right) \\
& =\sum_{i=1}^{2 n} a_{i}^{2} f\left(\gamma v_{i}\right)+\sum_{i \neq j} a_{i} a_{j} \omega\left(\gamma v_{i}, \gamma v_{j}\right) \\
& =f\left(\gamma\left(\sum_{i=1}^{2 n} a_{i} v_{i}\right)\right),
\end{aligned}
$$

since $f\left(\gamma v_{i}\right)=f\left(v_{i}\right)$ and $\gamma$ preserves $\omega$.

We claim that the bundle $\mathrm{Sp}_{2 n} \rightarrow \mathrm{Sp}_{2 n} / \mathrm{SO}_{2 n} \sim \mathbb{A}^{2 n}$ admits a multisection of degree $2^{2 n}$. Explicitly, it can be constructed as follows: fix an orthogonal basis $\left\{v_{1}, \ldots, v_{2 n}\right\}$ such that $f\left(v_{i}\right) \neq 0$, for $i=1, \ldots, 2 n$. We have an action of the affine group $\mathrm{B}=\mathbb{G}_{m} \rtimes \mathbb{G}_{a} \subset \mathrm{SL}_{2}$ given by

$$
\left(x_{i}, x_{n+i}\right) \mapsto\left(\lambda x_{i}, \mu x_{i}+\lambda^{-1} x_{n+i}\right), \text { for } i=1, \ldots, n .
$$

We claim that this gives a generically surjective map:

$$
\mathrm{SO}_{2 n} \times \mathrm{B}^{n} \rightarrow \mathrm{Sp}_{2 n}
$$

of degree $2^{2 n}$. The image of $\mathbf{B}^{n} \cdot\left\{f\left(v_{1}\right), \ldots, f\left(v_{2 n}\right)\right\}$ is dense in $\mathbb{A}^{2 n}$. Consider the intersection $\mathrm{B}^{n} \cap \mathrm{SO}_{2 n}$ :

$$
f\left(v_{i}\right)=\lambda^{2} f\left(v_{i}\right) \text {, and } f\left(v_{i+n}\right)=\mu^{2} f\left(v_{i}\right)+\lambda \mu \omega\left(v_{i}, v_{i+n}\right)+\lambda^{-2} f\left(v_{i+n}\right) .
$$

These equations can be solved in $k$, for each $i=1, \ldots, n$, and we have a dominant map $\mathrm{SO}_{2 n} \times \mathrm{B} \rightarrow \mathbb{A}_{i}^{2}$ of degree 4 , for each $i$. This concludes the proof. 


\section{Stable cohomology}

In this section we collect background material on the stable cohomology of finite groups, developing the theory over arbitrary algebraically closed fields $k$. We will omit $k$ from the notation when the field is clear from from the context.

For every finite group $G$ and a $G$-module $M$ we have the notion of group cohomology, as the derived functor $M \mapsto M^{G}$, the $G$-invariants, or, topologically, as the cohomology of the classifying space $B G=X / G$, where $X$ is a contractible space with a fixed point free action of $G$.

Passing to algebraic geometry, let $X$ be an algebraic variety over $k$, with an almost free action of a $G$. Let $X^{\circ} \subset X$ be the locus where the action is free. Let $M$ be a finite $G$-module. It defines a sheaf on $\tilde{X}:=X^{\circ} / G$. This gives a homomorphism from group cohomology of $G$ to étale cohomology of $\tilde{X}$ :

$$
H^{i}(G, M) \rightarrow H_{e t}^{i}(\tilde{X}, M) .
$$

Composing with restriction to the generic point we get a homomorphism

$$
\sigma_{i}^{*}: H^{i}(G, M) \rightarrow H^{i}\left(\mathcal{G}_{K}, M\right),
$$

where $\mathcal{G}_{K}$ is the absolute Galois group of the function field $K=k(\tilde{X})$. There are canonical isomorphisms

$$
H^{i}\left(\mathcal{G}_{K}, M\right)=\lim _{\vec{D}} H^{i}(X \backslash D, M),
$$

where the limit is taken over divisors of $D$. We can interpret elements in the kernel of $\sigma^{*}$ as classes vanishing on some Zariski open subvariety $\tilde{U} \subset \tilde{X}$.

REMARK 4.1. - Note that for fixed $G$ and $M$, the groups $\sigma_{i}^{*}\left(H^{i}(G, M)\right)=0$, for all $i>\operatorname{dim}(X)$, while the usual group cohomology need not vanish.

Proposition 4.2. - There exist a finite group $\tilde{G}$ and a sequence

$$
\mathcal{G}_{K} \stackrel{\tilde{\sigma}}{\longrightarrow} \tilde{G} \stackrel{\rho}{\longrightarrow} G
$$

of homomorphisms $\tilde{\sigma}$ and $\rho$ such that for all $0 \leq i \leq \operatorname{dim}(X)$ one has

$$
\operatorname{Ker}\left(\sigma_{i}^{*}\right) \subset \operatorname{Ker}\left(\rho_{i}^{*}\right),
$$

where $\rho_{i}^{*}: H^{i}(\tilde{G}, M) \rightarrow H^{i}(G, M)$ is the induced map on group cohomology. 
Proof. — The cohomology classes in $H^{i}\left(\mathcal{G}_{K}, \mathbb{Z} / \ell\right)$ are represented by continuous cocycles (in the natural topology on $\mathcal{G}_{K}$ ). Any element is induced from a finite group $H$. If it vanishes it also vanishes on a finite quotient $\tilde{G}$ of $\mathcal{G}_{K}$ and the maps $\mathcal{G}_{K} \rightarrow \tilde{G} \rightarrow G$ are continuous. Since the initial group $H^{i}(G, \mathbb{Z} / \ell)$ is finite there exists a $\tilde{G}$ where all elements from $H^{i}(G, \mathbb{Z} / \ell)$, which vanish on $\mathcal{G}_{K}$ are killed.

A special case of the above construction arises as follows: let $\varrho: G \rightarrow V$ be a faithful representation over an algebraically closed field $k$ and let $K=k(V)^{G}$ be the function field of the quotient. We have induced maps

$$
s_{i}^{*}: H^{i}(G, M) \rightarrow H^{i}\left(\mathcal{G}_{K}, M\right)
$$

and we can define the stable cohomology groups over $k$ :

$$
H_{k, s}^{i}(G, M):=H^{i}(G, M) / \operatorname{Ker}\left(s_{i}^{*}\right),
$$

which we will often identify with their image in $H^{i}\left(\mathcal{G}_{K}, M\right)$.

Proposition 4.3. - The cohomology groups $H_{k, s}^{i}(G, M)$

(1) do not depend on the representation;

(2) are functorial in $G$;

(3) are universal for $G$-actions: for any $G$-variety $X$ over $k$ the homomorphism $H^{i}(G, M) \rightarrow H^{i}\left(\mathcal{G}_{k(X)}, M\right)$ factors through $H_{k, s}^{i}(G, M)$;

(4) if $M$ is an $\ell$-torsion module, then

$$
H_{k, s}^{i}(G, M)=H_{k, s}^{i}\left(\mathrm{Syl}_{\ell}, M\right)^{\mathfrak{N}_{\ell}}
$$

where $\operatorname{Syl}_{\ell}=\operatorname{Syl}_{\ell}(G)$ is an $\ell$-Sylow subgroup of $G$ and $\mathfrak{N}_{\ell}=\mathfrak{N}_{\ell}(G)$ its normalizer in $G$.

Proof. - We apply Lemma 2.2. Choosing an appropriate Zariski open $G$ invariant subvariety $X^{\circ} \subset X$ we can reduce to the affine case, with free $G$ action. Let $V^{\circ} \subset V$ be a Zariski open subset where the action of $G$ is free. Put $\tilde{X}:=G \backslash X^{\circ}$ and $\tilde{V}:=G \backslash V^{\circ}$. We need to show that a class $\alpha \in H^{i}(G, M)$ whose image in $H^{i}\left(\mathcal{G}_{k(\tilde{V})}, M\right)$ is zero also vanishes in $H^{i}\left(\mathcal{G}_{k(\tilde{X})}, M\right)$. Such a class vanishes in $H_{e t}^{i}(\tilde{U}, M)$, where $\tilde{U} \subset \tilde{V}$ is an affine Zariski open subset. The preimage $U$ of $\tilde{U}$ in $V$ is a nonempty $G$-invariant affine Zariski open subset. Thus there exist an affine nonempty $G$-invariant Zariski open subset $U_{X} \subset X^{\circ}$ and a $G$-morphism $\phi_{U}: X^{\circ} \rightarrow V$ such that $\phi_{U}\left(U_{X}\right) \subset U$. This 
descends to a morphism $\tilde{X} \supset \tilde{U}_{X} \rightarrow \tilde{U} \subset \tilde{V}$. The image of $\alpha$ under the composition

$$
H^{i}(G, M) \rightarrow H_{e t}^{i}(\tilde{U}, M) \rightarrow H_{e t}^{i}\left(\tilde{U}_{X}, M\right) \rightarrow H_{e t}^{i}\left(\mathcal{G}_{k(\tilde{X})}, M\right)
$$

is zero. This proves (3). Applying this to $X=V^{\prime}$, for another faithful representation, we get (1).

Property (2) is proved as follows: First, let $H \subset G$ be a subgroup and $V$ a faithful $G$-representation. Consider the morphism $H \backslash V \rightarrow G \backslash V$. A class vanishing on a Zariski open subset of $G \backslash V$ also vanishes on a Zariski open subset of $H \backslash V$. Next, let $G \rightarrow H$ be a surjective homomorphism and $V_{G}$, resp. $V_{H}$, a faithful representation of $G$, resp. $H$. Then $W_{G}:=V_{H} \oplus V_{G}$ is a faithful representation of $G$ and we have a commutative diagram

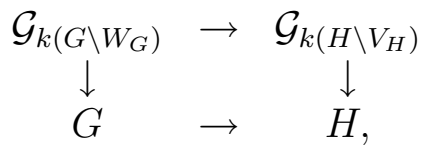

giving natural maps on cohomology.

We proceed with the proof of Property (4). Since $\ell$ and the cardinality of $G / \mathrm{Syl}_{\ell}$ are coprime, the map $\operatorname{Syl}_{\ell} \backslash V \rightarrow G \backslash V$ induces an invertible map on the cohomology of the open subvarieties of $G \backslash V$. The group $\mathfrak{N}_{\ell}(G) / \operatorname{Syl}_{\ell}(G)$ has order prime to $\ell$. The action of $\mathfrak{N}_{\ell}(G) / \operatorname{Syl}_{\ell}(G)$ on $M$ decomposes the module into a direct sum; so that

$$
H^{i}\left(\operatorname{Syl}_{\ell}(G), M\right)=H^{i}\left(\operatorname{Syl}_{\ell}(G), M\right)^{\mathfrak{N}_{\ell}(G)} \oplus R,
$$

so that the restriction of the trace map is zero on the module $R$.

We have

$$
H^{i}(G, M) \stackrel{\sim}{\longrightarrow} H^{i}\left(\operatorname{Syl}_{\ell}(G), M\right) \subset H^{i}\left(\operatorname{Syl}_{\ell}(G), M\right)^{\mathfrak{N}\left(\operatorname{Syl}_{\ell}(G)\right)} .
$$

Consider the image of $r \in R$ in $H_{k, s}^{*}\left(\operatorname{Syl}_{\ell}(G), M\right)$. We get a direct decomposition $H_{s}^{i}\left(\operatorname{Syl}_{\ell}(G), M\right)^{\mathfrak{N}\left(\operatorname{Syl}_{\ell}(G)\right)} \oplus R_{s}$, with $\operatorname{Tr}(r)=0$. Thus $H_{k, s}^{i}(G, M)$ surjects onto $H^{i}\left(\operatorname{Syl}_{\ell}(G), M\right)^{\mathfrak{N}\left(\operatorname{Syl}_{\ell}(G)\right)}$, and the map is an isomorphism.

LEMMA 4.4. - Let $V$ be a representation space for a faithful representation of group $G$ over an algebraically closed field $k$. Assume that $G \backslash V$ is isomorphic to affine space. Then any nontrivial element $\alpha \in H_{k, s}(G, \mathbb{Z} / \ell)$ has nontrivial restriction to the stable cohomology of a centralizer of a quasi-reflection in $G$. 
Proof. - If $\alpha \in H_{k, s}^{*}\left(k\left(\mathbb{A}^{n}\right), \mathbb{Z} / \ell\right)$ is nontrivial, then the residue of $\alpha$ is nontrivial on some irreducible divisor $D \subset \mathbb{A}^{n}=G \backslash V$ (see [6]). The preimage of $D$ in $V$ is a union of irreducible divisors $D_{1}, \ldots, D_{r}$. For each $i$, there exists a nontrivial $\gamma_{i} \in G$ acting trivially on all points of $D_{i}$. Thus each $D_{i}$ is a hyperplane in $V$. Hence $\gamma_{i}$ is a quasi-reflection.

Corollary 4.5. - Let $\mathcal{W}$ be a Weyl group. Then

$$
H_{k, s}^{i}(\mathcal{W}, \mathbb{Z} / \ell)=0, \quad \text { for all } i>0 \text { and all } \ell \neq 2 \text {. }
$$

We have

$$
H_{k, s}^{i}(\mathcal{W}, \mathbb{Z} / 2) \hookrightarrow \oplus_{\tau} H_{k, s}^{i}\left(\tau,(\mathbb{Z} / 2)^{r_{\tau}}\right),
$$

where $\tau$ runs over the set of 2-elementary abelian subgroups of $\mathcal{W}$, modulo conjugation, and $r_{\tau} \in \mathbb{N}$.

Proof. - The quasi-reflections in the standard faithful representation of $\mathcal{W}$ have order 2. Their centralizers are products of powers of $\mathbb{Z} / 2$ with smaller Weyl groups. It suffices to apply induction.

REMARK 4.6. - It is possible to obtain a more precise vanishing result following the approach for $\mathcal{W}=\mathfrak{S}_{n}$ in [9].

\section{Comparison with Serre's negligible classes}

Stable cohomology was defined by the first author in [2] and [3]. J.P. Serre, in his 1990-1991 course at College de France [14], defined a related but somewhat different notion.

In his terminology, negligible elements $\alpha \in H^{*}(G, M)$ are those which are killed under every surjective homomorphism $\mathcal{G}_{K} \rightarrow G$, where $K=k(\tilde{X})$ is the function field of a quotient $\tilde{X}=G \backslash X^{\circ}$. Negligible elements form an ideal in the total ring $H^{*}(G, M)$.

We are considering a smaller set of homomorphisms $\mathcal{G}_{K} \rightarrow G$, namely from Galois groups of fields of type $K=k(V)^{G}$, and the ideal of negligible classes defined by Serre is smaller. The resulting groups are different (for example, for $\mathbb{Z} / 2$-coefficients). The quotient ring obtained by Serre's construction surjects onto the ring $H_{k, s}^{*}(G, M)$, for any algebraically closed $k$. 


\section{Unramified cohomology}

Here we study unramified cohomology, which was introduced in [4] and [6] (see also [7]). Let $K=k(X)$ be a function field over an algebraically closed field $k$, and $M$ an étale sheaf on $X$. For every divisorial valuation $\nu \in \operatorname{Val}_{K}$ of $K$ we have a split exact sequence

$$
1 \rightarrow \mathcal{I}_{\nu} \rightarrow \mathcal{G}_{K_{\nu}} \rightarrow \mathcal{G}_{K_{\nu}} \rightarrow 1
$$

where $K_{\nu}$ is the completion of $K$ with respect to $\nu, \boldsymbol{K}_{\nu}$ the residue field and $\mathcal{I}_{\nu}$ is the inertia group. This gives an exact sequence in Galois cohomology

$$
H^{i}\left(\mathcal{G}_{K_{\nu}}, M^{\mathcal{I}_{\nu}}\right) \rightarrow H^{i}\left(\mathcal{G}_{K_{\nu}}, M\right) \stackrel{\delta_{\nu}}{\longrightarrow} H^{i-1}\left(\mathcal{G}_{K_{\nu}}, \mathcal{I}_{\nu} M\right)
$$

where $M^{\mathcal{I}_{\nu}}$, resp. $\mathcal{I}_{\nu} M$, are the sheaves of invariants, resp. coinvariants. Unramified cohomology is defined by

$$
H_{k, u n}^{i}\left(\mathcal{G}_{K}, M\right):=\cap_{\nu \in \operatorname{Val}_{K}} \operatorname{Ker}\left(\delta_{\nu}\right) \subset H^{i}\left(\mathcal{G}_{K}, M\right) .
$$

LemMA 6.1. - Let $\pi: X \rightarrow Y$ be a surjective morphism of algebraic varieties over $k$, and $M$ an étale sheaf on $Y$. Then there is a natural homomorphism:

$$
\pi^{*}: H_{k, \text { un }}^{i}(Y, M) \rightarrow H_{k, \text { un }}^{i}\left(X, \pi^{*}(M)\right) .
$$

Moreover, if $\pi$ is finite, then there is a natural homomorphism

$$
\pi_{*}: H_{k, u n}^{i}\left(X, \pi^{*}(M)\right) \rightarrow H_{k, u n}^{i}(Y, M)
$$

and the composition $\pi_{*} \circ \pi^{*}$ is multiplication by the degree of $\pi$.

Proof. - We have an embedding $\pi^{*}: k(X) \hookrightarrow k(Y)$ of function fields and a the corresponding map $\pi_{*}: \mathcal{G}_{k(Y)} \rightarrow \mathcal{G}_{k(X)}$ of Galois groups. A divisorial valuation $\nu$ of $k(Y)$ is either trivial on $\pi^{*}(k(X))$ or defines a divisorial valuation $\nu^{*}$ on $k(X)$. If $\nu$ is trivial on $\pi^{*}(k(X))$, then $\pi_{*}\left(\mathcal{I}_{\nu}\right)$ for the inertia subgroup $\mathcal{I}_{\nu} \subset \mathcal{G}_{k(Y)_{\nu}}$ and hence $\delta_{\nu}$ is zero on $\pi^{*} H^{*}\left(\mathcal{G}_{k(X)}, M\right)$. If $\nu$ on $\pi^{*}(k(X))$ coincides with $\nu^{*}$ then under the induced map $\pi_{*}: \mathcal{G}_{k(Y)_{\nu}} \rightarrow \mathcal{G}_{k(X)_{\nu^{\prime}}}$ we have $\pi_{*}\left(\mathcal{I}_{\nu}\right) \subset$ $\mathcal{I}_{\nu^{*}}$. Thus $\delta_{\nu^{*}}(\alpha)=0$ implies that $\delta_{\nu} \pi^{*}(\alpha)=0$ for all $\alpha \in H_{k, u n}^{*}\left(\mathcal{G}_{k(X)}, M\right)$.

Let $G$ be a finite group and $V$ a faithful $G$-representation as above. Let $K=k(V)^{G}$ be the function field of the quotient. We can consider its stable cohomology groups $H_{k, s}^{i}(G, M)$ as subgroups of $H^{i}\left(\mathcal{G}_{K}, M\right)$. Define unramified cohomology groups

$$
H_{k, \text { un }}(G, M):=H_{k, s}^{i}(G, M) \cap H_{k, u n}^{i}\left(\mathcal{G}_{K}, M\right) .
$$


Proposition 6.2. - Assume that $\operatorname{char}(k) \nmid|M|$. Then the unramified cohomology groups $H_{k, u n}^{i}(G, M)$

- do not depend on the representation $V$;

- are functorial in $G$.

Proof. - Let $V, V^{\prime}$ be two faithful representations of $G$ and consider the diagram:

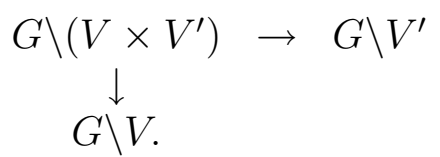

The case of constant coefficients follows from [8] and the observation that both arrows in the above diagram are natural vector bundles on the quotients. A small modification of the argument proves the claim for a general $G$-module $M$.

\section{General vanishing results}

In this section, we work over $k=\overline{\mathbb{F}}_{p}$. Here we collect general arguments proving the triviality of stable and unramified cohomology groups.

TheOREm 7.1. - Let $G$ be a finite group and $M$ a finite $p$-torsion $G$-module. Then, $H_{k, s}^{i}(G, M)=0$, for all $i>0$.

Proof. - See [15, Chapter 2, Proposition 3].

The main reason for introducing the unramified cohomology group is:

Theorem 7.2. - Let $V$ be a faithful representation of $G$. If $K=k(V)^{G}$ is a purely transcendental extension of $k$, then, for all $i>0$, we have

$$
H_{k, u n}^{i}(G, \mathbb{Z} / \ell)=0 .
$$

Proof. - Immediate from [6, Corollary 1.2.1].

TheOREM 7.3 (Lang). - Let $\mathrm{G}$ be an algebraic group over $k$. Let $F$ be an automorphism of $\mathrm{G}(k)$ which is a composition of an element in $\operatorname{Aut}(\mathrm{G})(k)$ and a Frobenius of $k=\overline{\mathbb{F}}_{p}$. Let $G=G^{F} \subset \mathrm{G}(k)$ be the finite subgroup fixed by $F$. Then $G \backslash \mathrm{G} \simeq \mathrm{G}$, hence is a rational variety. 
Proof. - Consider the map

$$
\begin{aligned}
\tau: \mathrm{G} & \rightarrow \mathrm{G} \\
x & \rightarrow F(x)^{-1} x .
\end{aligned}
$$

The action of $\tau$ on the Lie algebra of $\mathrm{G}$ is surjective with kernel a finite subgroup $G=G^{F}$. Note that $\tau$ coincides with the composition

$$
\tau: \mathrm{G} \rightarrow G \backslash \mathrm{G} \rightarrow \mathrm{G} .
$$

Indeed, if $\tau(x)=\tau(y)$, then $F(x)^{-1} x=F(y)^{-1} y$, or $F\left(x y^{-1}\right)^{-1} x y^{-1}=1$, or $x y^{-1} \in G$. If follows that $x=g y, g \in G$. The converse is clear. Thus $G \backslash G$ is rational.

Lemma 7.4. - Let $\ell$ be a prime and $\pi: X \rightarrow Y$ a separable morphism $k$ varieties of finite degree prime to $\ell$. Assume that $H_{k, u n}^{i}(X, \mathbb{Z} / \ell)=0$. Then $H_{k, u n}^{i}(Y, \mathbb{Z} / \ell)=0$.

Proof. - Immediate from Lemma 6.1: the $\operatorname{degree} \operatorname{deg}(\pi)$ is prime to $\ell$ and multiplication by $\operatorname{deg}(\pi)$ is invertible on $H_{k, u n}^{i}(Y, \mathbb{Z} / \ell)$.

This lemma will be applied to $Y=G \backslash V$. The goal will be to construct $X$ with vanishing unramified cohomology.

Corollary 7.5. - Let $G=G^{F} \subset \mathrm{G}(k)$ be as above. Let $X$ be a G-linear variety over $k$. Assume that there exist a variety $Y$ over $k$, with trivial $G$ action, and a G-equivariant finite morphism

$$
\pi: \mathrm{G} \times Y \rightarrow X .
$$

Let $S$ be the set of all primes dividing the degree of $\pi$. Then

$$
H_{k, u n}^{i}(G, \mathbb{Z} / \ell)=0, \text { for all } i>0 \text { and } \ell \notin S \cup\{p\} \text {. }
$$

Proof. - Any affine connected algebraic group over $k=\overline{\mathbb{F}}_{p}$ is rational. By Lang's theorem, the quotient $G \backslash \mathrm{G}$ is isomorphic to $\mathrm{G}$, and thus rational. For primes $\ell \notin S \cup\{p\}$ not dividing the degree of $\pi$, the induced map on cohomology is injective. This concludes the proof.

TheOREM 7.6. - Let $\mathrm{G}$ be a Lie group over $k$. Let $G=G^{F} \subset \mathrm{G}(k)$ be a finite subgroup. Put

$$
s(G):= \begin{cases}\{p, 2\} & \text { for } G \text { of type } C \text { or } D_{n}, n \geq 5 \\ \{p, 2,3\} & \text { for } G \text { of type } D_{4}, F_{4}, E_{6}, E_{7} \\ \{p\} & \text { otherwise }\end{cases}
$$


Then

$$
H_{k, \text { un }}^{i}(G, \mathbb{Z} / \ell)=0
$$

for all $\ell \notin s(G)$.

We have a natural homomorphism

$$
H^{i}(G, \mathbb{Z} / \ell) \rightarrow H^{i}(G \backslash \mathrm{G}, \mathbb{Z} / \ell) .
$$

By Lang's theorem 7.3, $G \backslash \mathrm{G} \simeq \mathrm{G}$, as algebraic varieties. Thus we get a homomorphism

$$
\rho: H^{i}(G, \mathbb{Z} / \ell) \rightarrow H_{e t}^{i}(\mathrm{G}, \mathbb{Z} / \ell) .
$$

Assume that $G$ is semi-simple. Then $\operatorname{Pic}(G) \simeq \pi_{1}(G)$ is a finite group and $\mathrm{G}=\tilde{\mathrm{G}} / \operatorname{Pic}(G)$, where $\tilde{\mathrm{G}}$ is the universal cover of $\mathrm{G}$. We obtain a natural homomorphism

$$
\eta: H_{e t}^{i}(\operatorname{Pic}(\mathrm{G}), \mathbb{Z} / \ell) \rightarrow H_{e t}^{i}(\mathrm{G}, \mathbb{Z} / \ell) .
$$

TheOREM 7.7. - Let $\mathrm{G}$ be a semi-simple Lie group over $k$. Let $G=G^{F} \subset$ $\mathrm{G}(k)$ be a finite subgroup. Consider the diagram

$$
\begin{array}{r}
H_{e t}^{i}(\operatorname{Pic}(\mathrm{G}), \mathbb{Z} / \ell) \\
\sigma_{*}^{i} \circ \eta
\end{array}
$$

Then the image of $\rho$ is contained in the image of $\eta$.

Proof. - Standard computation using restriction of the fibration $G \rightarrow G / T$ to $\mathrm{G}^{\circ}=\mathrm{T} \times \mathbb{A}^{N}$ and the transgression homomorphism. Geometrically, the map $\sigma^{*} \circ \nu$ in the diagram corresponds to the embedding of the maximal Bruhat cell $\mathrm{U}^{+} \mathrm{TU}^{-}$into the $G \backslash \mathrm{G}=\mathrm{G}$, where $\mathrm{T}$ is the maximal torus. We have

$$
H_{k, s}^{*}(\mathrm{~T}, \mathbb{Z} / \ell)=H^{*}(\mathrm{~T}, \mathbb{Z} / \ell),
$$

for $\ell \neq p$, and these coincide with $H^{*}(\operatorname{Pic}(\mathrm{T}) \otimes \mathbb{Z} / \ell, \mathbb{Z} / \ell)$.

Corollary 7.8. - Assume that $\mathrm{G}$ is simply-connected and that the natural translation action of $\mathrm{G}$ on itself is affine. Then

$$
H_{k, s}^{i}(\mathrm{G}, \mathbb{Z} / \ell)=0,
$$

for all $i>0$ and $\ell \nmid q$. 
Proof. - For simply-connected $\mathrm{G}$ the image of $H^{*}(\mathrm{G}, \mathbb{Z} / \ell)$ in $H_{k, s}^{*}(\mathrm{~T}, \mathbb{Z} / \ell)$ is trivial. This follows from the computation of the transgression homomorphism for $G / T$, since $G$ is a $T$-torsor over $G / T$, with $\operatorname{Pic}(G / T)$ isomorphic to the character group of the maximal torus $T$. Geometrically, this corresponds to the fact that $T$ is contractible in $G$, provided $G$ is simply-connected.

Corollary 7.9. - Let $\mathrm{G}$ be a semi-simple Lie group over $k$ and $G \subset \mathrm{G}(k)$. Then the image of $H_{k, u n}^{i}(G)$ in $H_{k, s}^{i}\left(G \backslash \mathrm{G}(k) / G, \mathbb{Z}_{\ell}\right)$ is trivial.

Proof. - The group $G$ is contained in a subgroup $G^{F}$ of $\mathrm{G}(k)$, for some $F$, and $G=\left[\mathrm{G}(k)^{F}, \mathrm{G}(k)^{F}\right]$ in this case, with $G^{F} / G$ a group of order coprime to $p$. As before $G^{F} \backslash \mathrm{G}=\mathrm{G}$ as an algebraic variety, which contains the cell $\mathrm{U}^{-} \mathrm{TU}^{+}$. Thus $G \backslash G$ contains a finite abelian unramified covering of $\mathrm{U}^{-} \mathrm{TU}^{+}$which is also a product $\mathrm{U}^{-} \tilde{\mathrm{T}} \mathrm{U}^{+}$, where $\tilde{\mathrm{T}}$ is a torus. The cohomology $H_{k, s}^{i}(G \backslash \mathrm{G}, \mathbb{Z} / \ell)$ embedds into the cohomology of $\tilde{\mathrm{T}}$. Hence $H_{k, u n}^{i}(G, \mathbb{Z} / \ell)$ map to zero.

\section{Reduction to Sylow subgroups}

Let $G$ be a finite group. For $H \subset G$ let $\mathfrak{N}_{G}(H)$ denote the normalizer of $H$. Let $\operatorname{Syl}_{\ell}(G)$ be an $\ell$-Sylow subgroup of $G$. Recall the following classical result (see, e.g., [1, Section III.5]):

$$
H^{i}(G, \mathbb{Z} / \ell)=H^{i}\left(\operatorname{Syl}_{\ell}(G), \mathbb{Z} / \ell\right)^{\mathfrak{N}_{G}\left(\operatorname{Syl}_{\ell}(G)\right)} .
$$

TheOREM 8.1. - Let $G$ be a finite group. Let $\ell$ be a prime distinct from the characteristic of $k$. Then there is an isomorphism

$$
H_{k, s}^{i}(G, \mathbb{Z} / \ell) \stackrel{\sim}{\longrightarrow} H_{k, s}^{i}\left(\operatorname{Syl}_{\ell}(G), \mathbb{Z} / \ell\right)^{\mathfrak{N}_{G}\left(\operatorname{Syl}_{\ell}(G)\right)} .
$$

Similarly,

$$
H_{k, \text { un }}^{i}(G, \mathbb{Z} / \ell) \stackrel{\sim}{\longrightarrow} H_{k, \text { un }}^{i}\left(\operatorname{Syl}_{\ell}(G), \mathbb{Z} / \ell\right)^{\mathfrak{N}_{G}\left(\operatorname{Syl}_{\ell}(G)\right)} .
$$

Proof. - Let $V$ be a faithful representation of $G$ over $k$. Then the map

$$
\pi: \operatorname{Syl}_{\ell}(G) \backslash V \rightarrow G \backslash V
$$

is a finite, separable and surjective map of degree prime to $\ell$. Hence $\pi_{*} \circ \pi^{*}$ is invertible in cohomology. This implies the first claim.

The fact that the local ramification indices of $\pi$ are coprime to $\ell$ implies the second claim. 
LEMmA 8.2. - Let $G \subset \mathrm{G}(k)$ be a finite subgroup of a Lie group $\mathrm{G}$ over $k$ which has a trivial intersection with the center of $\mathrm{G}(k)$. Let $G^{\text {int }} \backslash \mathrm{G}$ be the quotient of $\mathrm{G}$ by the conjugation action of $G$. Then the homomorphisms

$$
h^{\text {int }}: H_{k, s}^{i}(G, \mathbb{Z} / \ell) \rightarrow H_{k, s}^{i}\left(G^{i n t} \backslash \mathrm{G}, \mathbb{Z} / \ell\right)
$$

and

$$
h_{u n}^{\text {int }}: H_{k, u n}^{i}(G, \mathbb{Z} / \ell) \rightarrow H_{k, \text { un }}^{i}\left(G^{i n t} \backslash \mathrm{G}, \mathbb{Z} / \ell\right)
$$

are injective.

Proof. - The conjugation action of $G$ on $\mathrm{G}$ is almost free, and the maps $h^{\text {int }}, h_{u n}^{\text {int }}$ are well-defined. The identity $e \in G(k)$ is a smooth invariant point for the conjugation action. In particular, the local (conjugation) action of any $\ell$-Sylow subgroup of $G$ lifts to characteristic 0 with the same invariant point. The action of $\operatorname{Syl}_{\ell}(G), \ell \neq p$, in a formal neighborhood of $e$ is equivalent to the linear action on the tangent space $\left.T \mathrm{G}\right|_{e}$ at $e$ and lifts to a linear representation of $\operatorname{Syl}_{\ell}(G)$ in characteristic 0. Since the linear action of $G$ on the linear space $\left.T \mathrm{G}\right|_{e}$ over $k$ is equivariant with respect to the locally free action of $\operatorname{Syl}_{\ell}(G)$ on $\left.T \mathrm{G}\right|_{e}$, any element of $H^{i}\left(\operatorname{Syl}_{\ell}(G), \mathbb{Z} / \ell\right)$ which is trivial in the quotient of the formal neighborhood of $0 \in \hat{H}_{k, s}^{i}\left(\left.T G\right|_{e} / \operatorname{Syl}_{\ell}(G), \mathbb{Z} / \ell\right)$ will also be trivial in $H_{k, s}^{i}\left(\left.T \mathrm{G}\right|_{e} / \operatorname{Syl}_{\ell}(G), \mathbb{Z} / \ell\right)=H_{k, s}^{i}(G, \mathbb{Z} / \ell)$.

This proves the injectivity of $h^{i n t}$. The same argument works for $h_{u n}^{i n t}$.

Lemma 8.3. - Let $G, H$ be finite groups. Let $\rho: H \rightarrow W$ be a faithful $k$ representation of $H$. Assume that $H \backslash W$ is stably rational. Let $U$ be a faithful representation of $\tilde{G}:=H 2_{S} G$, where $S$ is a finite $G$-set. Then $\tilde{G} \backslash U$ is stably birationally equivalent to $G \backslash V$, where $V$ is a faithful representation of $G$.

Proof. - Put

$$
\rho_{S}=\oplus_{s \in S} \rho_{s}: H_{S}:=\prod_{s \in S} H_{s} \rightarrow \operatorname{Aut}\left(W_{S}\right), \quad W_{S}=\oplus_{s \in S} W_{s},
$$

where $H_{s}=H, W_{s}=W$, for all $s \in S$, and $\rho_{s}=\rho$ on the factor $H_{s}$ and trivial on $H_{s^{\prime}}$, for $s^{\prime} \neq s$. We construct $U:=V^{\prime} \oplus W_{S}$, where $V^{\prime}$ is a faithful representation of $G$ and extend the action of $G$ to $V_{S}$ via the $G$-action on $S$. This gives a representation of $\tilde{G}=H 2_{S} G$ in $\operatorname{Aut}(U)$. The quotient space is a fibration over $G \backslash V^{\prime}$ with fibers $(H \backslash W)^{|S|}$. We can assume that $H \backslash W$ is rational. The action of $G$ on $(H \backslash W)^{|S|}$ permutes the coordinates. It follows that $\tilde{G} \backslash U$ is birationally equivalent to a vector bundle over $G \backslash V^{\prime}$. 
Corollary 8.4. - Let $G=\operatorname{Syl}_{\ell}\left(\mathfrak{S}_{n}\right)$ and let $V$ be a faithful representation of $G$. Then $G \backslash V$ is stably rational.

Proof. - The $\ell$-Sylow subgroups of $\mathfrak{S}_{n}$ are products of wreath products of groups $\mathbb{Z} / \ell \imath \cdots\urcorner \mathbb{Z} / \ell$ (see [1, VI.1]). The quotient $H \backslash W$ is rational, for a faithful representation $W$ of $H=\mathbb{Z} / \ell$. We apply induction to conclude that the quotient $G \backslash V$ is stably rational.

Corollary 8.5. - Let $G=\operatorname{Syl}_{\ell}\left(\mathrm{GL}_{n}\left(\mathbb{F}_{q}\right)\right)$, with $\ell \nmid q$, and let $V$ be a faithful representation of $G$. Then $G \backslash V$ is stably rational.

Proof. - The structure of $\ell$-Sylow subgroups of $\mathrm{GL}_{n}\left(\mathbb{F}_{q}\right)$ is known (see [1, VII.4]: it is also a product of iterated wreath products of cyclic $\ell$-groups. Thus we can apply Lemma 8.3.

COROLlary 8.6. - Let $k$ be an algebraically closed field of characteristic zero. Then

$$
H_{k, u n}^{i}\left(\mathrm{GL}_{n}\left(\mathbb{F}_{q}\right), \mathbb{Z} / \ell\right)=0 \quad \text { for all } i>0, \text { and } \ell \nmid q .
$$

REMARK 8.7. - Similar computations can be performed for some other finite groups of Lie type, e.g., for $\mathrm{O}_{2 m}^{ \pm}\left(\mathbb{F}_{q}\right)$ and $\operatorname{Sp}_{n}\left(\mathbb{F}_{q}\right)$.

\section{References}

[1] A. Adem And R. J. Milgram, Cohomology of finite groups, second ed., Grundlehren der Mathematischen Wissenschaften, vol. 309, SpringerVerlag, Berlin, 2004.

[2] F. Bogomolov - "Bloch's conjecture for torsion cohomology of algebraic varieties", 1985, Amsterdam University preprint 85-20.

[3] _ "Stable cohomology", 1986, Arbeitstagung talk, MPI preprint.

[4] _ "The Brauer group of quotient spaces of linear representations", Izv. Akad. Nauk SSSR Ser. Mat. 51 (1987), no. 3, p. 485-516, 688.

[5] F. Bogomolov, J. Maciel and T. Petrov - "Unramified Brauer groups of finite simple groups of Lie type $A_{l}$ ", Amer. J. Math. 126 (2004), no. 4, p. 935-949.

[6] J.-L. Colliot-Thélène and M. Ojanguren - "Variétés unirationnelles non rationnelles: au-delà de l'exemple d'Artin et Mumford", Invent. Math. 97 (1989), no. 1, p. 141-158. 
[7] J.-L. Colliot-ThÉlène, - "Birational invariants, purity and the Gersten conjecture, $K$-theory and algebraic geometry: connections with quadratic forms and division algebras (Santa Barbara, CA, 1992), Proc. Sympos. Pure Math. 58, Part 1, Amer. Math. Soc., Providence, RI, 1995. p. 1-64.

[8] J.-L. Colliot-Thélène and J.-J. SAnsuC - "The rationality problem for fields of invariants under linear algebraic groups (with special regards to the Brauer group)", Algebraic groups and homogeneous spaces, Tata Inst. Fund. Res. Stud. Math., Tata Inst. Fund. Res., Mumbai, 2007, p. $113-186$.

[9] S. Garibaldi, A. Merkurjev and J.-P. Serre - Cohomological invariants in Galois cohomology, University Lecture Series, vol. 28, American Mathematical Society, Providence, RI, 2003.

[10] B. Kunyavski - "The Bogomolov multiplier of finite simple groups", 2008, this volume.

[11] E. PEYRE - "Unramified cohomology and rationality problems", Math. Ann. 296 (1993), no. 2, p. 247-268.

[12] __ "Unramified cohomology of degree 3 and Noether's problem", Invent. Math. 171 (2008), no. 1, p. 191-225.

[13] D. J. Saltman - "Noether's problem over an algebraically closed field", Invent. Math. 77 (1984), no. 1, p. 71-84.

[14] J.-P. SERRE - "Course at College de France 1990-1991".

[15] _ Cohomologie galoisienne, fifth ed., Lecture Notes in Mathematics, vol. 5, Springer-Verlag, Berlin, 1994.

[16] J. Tits - "Sur les degrées des extensions de corps déployant les groupes algébriques simples", C. R. Acad. Sci. Paris Sr. I Math. 315 (1992), no. 11, p. 1131-1138. 